\title{
Replacing sedentary time with physical activity: a I5-year follow-up of mortality in a national cohort
}

This article was published in the following Dove Press journal:

Clinical Epidemiology

\author{
Ing-Mari Dohrn' \\ Lydia Kwak ${ }^{2}$ \\ Pekka Oja ${ }^{3}$ \\ Michael Sjöström ${ }^{4}$ \\ Maria Hagströmer ${ }^{1,5}$ \\ 'Department of Neurobiology, \\ Care Sciences and Society (NVS), \\ ${ }^{2}$ Institute of Environmental Medicine \\ (IMM), Karolinska Institutet, \\ Stockholm, Sweden; ${ }^{3}$ UKK Institute, \\ Tampere, Finland; ${ }^{4}$ Department of \\ Biosciences and Nutrition (BioNut), \\ Karolinska Institutet, ${ }^{5}$ Functional \\ Area Occupational Therapy and \\ Physiotherapy, Karolinska University \\ Hospital, Stockholm, Sweden
}

Background: Sedentary behavior is associated with health risks in adults. The potential benefits of reducing sedentary time may be dependent not only on decrease per se, but also on the type of activity it replaces. Few longitudinal studies have investigated the effects on mortality when replacing objectively assessed sedentary time with another physical activity (PA) behavior.

Objective: To investigate the effects of replacing objectively assessed sedentary time with time in light-intensity PA or moderate-vigorous PA (MVPA) on all-cause mortality, cardiovascular disease (CVD) mortality or cancer mortality in a cohort with 15 years follow-up time.

Methods: In total, 851 women and men from the population-based Sweden Attitude Behaviour and Change study were included. Time spent sedentary, in light-intensity PA and in MVPA were assessed using an Actigraph 7164 accelerometer. Mortality data were obtained from Swedish registers. Cox proportional hazards models estimated hazard ratios (HR) of mortality with $95 \%$ confidence intervals (CI) and isotemporal substitution models were used to estimate the effect of replacing sedentary behavior with PA for the same amount of time.

Results: Over a follow-up of 14.2 years (SD 1.9) with 12,117 person-years at risk, 79 deaths occurred, 24 deaths from CVD, 27 from cancer, and 28 from other causes. Replacing 30 minutes/day of sedentary time with light-intensity PA was associated with significant reduction in all-cause mortality risk (HR: 0.89, 95\% CI: 0.81-0.98) and CVD mortality risk (HR: 0.76, 95\% CI: 0.63-0.92). Replacing 10 minutes of sedentary time with MVPA was associated with reduction in CVD mortality risk (HR: $0.62,95 \%$ CI: $0.42-0.91$ ). No statistically significant reductions were found for cancer mortality.

Conclusion: This statistical modelling study suggests that replacing sedentary time with light-intensity PA could have beneficial effect on both all-cause mortality and CVD mortality. Replacing sedentary time with MVPA could reduce CVD mortality.

Keywords: accelerometry, isotemporal substitution, light intensity, moderate-to-vigorous intensity, prospective, sedentary behavior

\section{Introduction}

There is a growing body of evidence that sedentary behavior is associated with a multitude of serious health risks in adults. ${ }^{1,2}$ Studies have found that excessive amounts of time spent sedentarily is a risk factor for mortality, type 2 diabetes, metabolic syndrome, cardiovascular disease (CVD), and cancer. ${ }^{1-5}$ Sedentary behavior is defined as any waking behavior characterized by an energy expenditure $\leq 1.5$ metabolic equivalent of task while in a sitting, reclining, or lying posture. ${ }^{6}$ It has been shown that mortality risk increases above 7.5 hours/day of sedentary time, with high risk above 10 hours. ${ }^{3,7}$ In a previous study, we found that individuals who spent 10 hours/day sedentarily
Correspondence: Ing-Mari Dohrn Department of Neurobiology, Care Sciences and Society (NVS), Karolinska Institutet, Stockholm SE-I7I 77, Sweden Tel +46708444 188

Email ing-mari.dohrn@ki.se 
had $>2.5$ times higher risk of death compared to those who spent 6.5 hours/day sedentarily. ${ }^{8}$ Evidence is inconclusive whether the increased mortality risk is independent of time in moderate-vigorous PA (MVPA), 2,4,9,10 and there is a need for research that can contribute to differentiating the effects of low PA levels from being sedentary per se. The average amount of daily time spent in light-intensity PA (1.6-2.9 metabolic equivalent of task), such as activities of everyday living, is about the same as time spent in sedentary behaviors. ${ }^{11}$ This suggests huge potential for replacing time spent in sedentary activities with light-intensity PA.

Most longitudinal studies that have found sedentary behavior to be a risk factor for early death have used selfreported data, ${ }^{2,12}$ subject to low accuracy due to recall bias or social desirability. ${ }^{13,14}$ These limitations can be overcome by the use of new technology, such as wearable activity monitors, providing objective assessment of sedentary time and PA with considerable precision. However, the literature is still limited regarding objectively assessed sedentary time and mortality in general, and very few longitudinal studies to date have studied the effects on mortality when replacing one PA behavior with another. ${ }^{15,16}$ Because total time in a day is finite, sedentary time, light-intensity PA, and MVPA are interdependent, and a decrease in time spent in one PA intensity means increased time spent in another. The potential benefits of reducing sedentary time may thus be dependent not only on decrease per se but also on the type of activity it replaces. These effects can be explored by isotemporal substitution modeling, originally developed from nutritional epidemiology and recently introduced in PA research. ${ }^{17}$ This statistical approach offers more specific insights into the association of different time-dependent activity classes with mortality compared to traditional nonsubstitutional approaches. In this study, we used data from a Swedish longitudinal cohort study with 15 years of follow-up to investigate the theoretical consequences of replacing sedentary time with the same duration of light-intensity PA or with MVPA on all-cause mortality, CVD mortality, and cancer mortality.

\section{Materials and methods \\ Data collection}

This study used data from the Sweden Attitude Behaviour and Change (ABC) study of 2001-2002, a nationally representative sample of Swedish adults aged 18-75 years. In total, 3,300 (52\% women) people were contacted, 2,262 (54\% women) were eligible, and 1,221 (55\% women) provided objective PA data. ${ }^{18,19}$ For the present study, we included those 851 persons ( $56 \%$ women) who were $\geq 50$ years at follow-up in 2015. The ABC data collected in 2001-2002 were combined with register data of mortality of 2002-2015 collected from the National Board of Health and Welfare's Cause of Death Register and the Swedish Cancer Registry in 2016. The Cause of Death Register contains both cause-ofdeath data and underlying causes of death coded according to the international version of the disease classification ICD10.

\section{Sedentary time and PA}

Sedentary time and PA were assessed with the uniaxial ActiGraph (Pensacola, FL, USA) 7164 accelerometer. The ActiGraph is a small, lightweight electronic device capturing time-varying acceleration in the vertical axis specified in an arbitrary unit named counts that can be translated to intensity and duration of PA. The sampling was set at counts per minute. The accelerometers were mailed together with a questionnaire and instructions to wear the accelerometer on the lower back, using an accompanying elastic belt, for seven consecutive days except during sleep and water-based activities, and then to return the accelerometer by prepaid mail.

Nonwearing time was defined as an interval of at least 60 consecutive minutes of zero counts, with allowance for up to 2 minutes of $1-100$ counts. ${ }^{20}$ Participants providing at least one valid day of accelerometer data defined as $>10$ hours of wearing time were included. ${ }^{13,18}$ Cutoff points to estimate the time spent sedentarily and in different PA intensities were defined in accordance with previous population-based studies: sedentary time was defined as $<100$ counts/minute, light-intensity PA as 100-2,019 counts/minute, and MVPA as $\geq 2,020$ counts/minute. ${ }^{4,13,21}$ The amount of time spent in vigorous activities was too small to analyze separately. ${ }^{18}$

\section{Covariates}

Demographic and anthropometric data were self-reported and obtained from a baseline questionnaire delivered by mail. ${ }^{18}$ Covariates included in this study were age (years, continuous), sex, body mass index (BMI) calculated from reported height and weight, smoking status (never/former or current), history of chronic disease - hypertension, heart disease, diabetes, or cancer (yes/no) - and education (less than high school, high school/equivalent diploma, or university degree). The study was approved by the regional ethical review board at the Karolinska Institutet (Dnr 378/02, 2012/707 31/1, 2015 1578/32).

\section{Statistical analyses}

Cox proportional-hazard models were applied to estimate HRs of mortality with $95 \%$ CIs. Follow-up extended from the first day of accelerometer assessment until the date of death or censoring on December 31, 2015, whichever occurred 
first. All covariates were examined for potential confounding or interaction by performing separate statistical tests for each covariate. Schönfeld residuals were examined to assess proportional-hazard assumption. ${ }^{22}$ Final models were stratified for sex and age tertiles, since these variables did not meet the proportional-hazard assumption, and included the covariates; education, smoking status, and chronic disease.

Isotemporal substitution models were used to investigate the theoretical consequences of replacing one PA behavior with another PA behavior for the same amount of time. This method allows the interdependence of daily activities of different intensity to be taken into account. We recalculated the PA variables from continuous 1-minute variables to 10and 30-minute variables. This way, we were able to estimate the consequences of replacing both a 10 -minute bout and a 30 minute bout of sedentary time with light-intensity PA or MVPA, respectively. Light-intensity PA and MVPA were entered into the models simultaneously, along with a total accelerometer-wearing time variable and covariates, but excluding sedentary time. By including the total accelerometer-wearing time variable in the model, time was isotemporal, and the HR for light-intensity PA and MVPA reflected the effect of substituting a bout of sedentary time with an equal time bout of the specific PA variable. The isotemporal modeling of PA was first described by Mekary et al. ${ }^{17} P$-values $\leq 0.05$ were considered statistically significant. Statistical analyses were computed in Stata version 11.0 (StataCorp LP, College Station, TX, USA).

\section{Results}

Descriptive statistics of the total study population and stratified by tertiles of sedentary time are shown in Table 1. The average age at follow-up was 66.7 (SD 10.2) years, range 50-89 years. The major part of daily activities was sedentary time, ranging 3-13 hours per day with an average of 8 hours 12 minutes per day for the total sample. The average time spent in light-intensity PA was 5 hours 44 minutes per day, and 31 minutes per day in MVPA. Over a follow-up of 14.2 (SD 1.9) years with 12,117 person-years at risk, 79 deaths (32 women, 47 men) occurred: 24 deaths from CVD, 27 from cancer, and 28 from other causes (Table 2 ). The crude, unweighted death rate was 0.65 per 100 person-years. No deaths occurred within the first year from inclusion, and no deaths were coded as accidental.

Figure 1 shows the theoretical consequences of replacing sedentary time in 10- and 30-minute bouts with an equal amount of light-intensity PA or MVPA. Isotemporal substitution analyses with fully adjusted models indicated $11 \%$ and $24 \%$ lower risk of all-cause mortality and CVD mortality, respectively, when 30 minutes/day of sedentary

Table I Baseline characteristics of the total study population and stratified by tertiles of sedentary time

\begin{tabular}{|c|c|c|c|c|}
\hline \multirow[t]{2}{*}{ Characteristics } & \multirow[t]{2}{*}{ Total } & \multicolumn{3}{|c|}{ Sedentary time (min/day) } \\
\hline & & $\leq 454$ & $>454-\leq 528$ & $>528$ \\
\hline $\mathrm{n}$ & 851 & 283 & 284 & 284 \\
\hline Age (years) & $52.8(10.2)$ & $51.2(10)$ & $54.6(10.5)$ & $52.5(10)$ \\
\hline Women (\%) & 55.9 & 60.8 & 58.4 & 55.9 \\
\hline BMI $\left(\mathrm{kg} / \mathrm{m}^{2}\right)$ & $25.6(3.6)$ & $25.3(3.5)$ & $25.7(3.5)$ & $25.8(3.6)$ \\
\hline Smoker (\%) & 26 & 25 & 24.3 & 27.6 \\
\hline \multicolumn{5}{|l|}{ Chronic disease (\%) } \\
\hline Any & 19 & 15.1 & 19.8 & 22.1 \\
\hline Hypertension & 14 & 10.4 & 15.6 & 16.1 \\
\hline Heart disease & 3.5 & 3.2 & 2.1 & 5 \\
\hline Diabetes & 2.5 & I.I & 2.1 & 4.3 \\
\hline Cancer & 2.7 & 2.8 & 2.8 & 2.5 \\
\hline \multicolumn{5}{|l|}{ Education (\%) } \\
\hline Less than high school & 30.6 & 34.1 & 31.3 & 26.2 \\
\hline High school & 38.4 & 38.4 & 38.7 & 37.9 \\
\hline University & 31 & 27.4 & 29.9 & 35.8 \\
\hline LIPA (min/day) & 344.4 (93.2) & $415.4(82.3)$ & $337.8(70.8)$ & $280.2(71.6)$ \\
\hline MVPA (min/day) & $3 \mid .4(3 \mid .2)$ & $4 I .4(44.6)$ & $29.6(21.5)$ & $23.2(17.5)$ \\
\hline Counts/day $(\times 1,000)$ & $312.6(213.8)$ & $410.4(311.6)$ & $293.7(120.1)$ & $234(100.3)$ \\
\hline Wearing time (h/day) & I $4.5(1.3)$ & $14.2(1.4)$ & I $4.3(\mathrm{I} .2)$ & $14.9(1.2)$ \\
\hline \multicolumn{5}{|l|}{ Valid days (\%) } \\
\hline 7 & 67 & 62.2 & 65.5 & 73.2 \\
\hline $3-6$ & 31.2 & 36.7 & 32.4 & 24.6 \\
\hline$\leq 3$ & 1.8 & I.I & 2.1 & 2.1 \\
\hline
\end{tabular}

Note: Data expressed as mean (SD) or percentages.

Abbreviations: BMI, body mass index; LIPA, light-intensity physical activity; MVPA, moderate-vigorous physical activity. 
Table 2 Baseline characteristics of participants who died during follow-up

\begin{tabular}{|c|c|c|c|c|}
\hline \multirow[t]{2}{*}{ Characteristics } & \multirow[t]{2}{*}{ All-cause } & \multicolumn{3}{|c|}{ Cause of death } \\
\hline & & CVD & Cancer & Other \\
\hline $\mathrm{n}$ & 79 & 24 & 27 & 28 \\
\hline Age (years) & $77.5(8)$ & $81.2(6.6)$ & $74.1(8.9)$ & $77.6(6.9)$ \\
\hline Women (\%) & 40.5 & 25 & 48.1 & 46.4 \\
\hline BMI $\left(\mathrm{kg} / \mathrm{m}^{2}\right)$ & $26.2(3)$ & $26.8(3.2)$ & $26(3)$ & $25.5(3.6)$ \\
\hline Smoker (\%) & 26.6 & 25 & II.I & 32.1 \\
\hline \multicolumn{5}{|l|}{ Chronic disease (\%) } \\
\hline Any & 24 & 33.3 & 19.8 & 32.1 \\
\hline Hypertension & 8.8 & 12.5 & 0 & 14.2 \\
\hline Heart disease & 8.8 & 16.7 & 0 & 10.7 \\
\hline Diabetes & 5.1 & 8.3 & 0 & 7.1 \\
\hline Cancer & 6.3 & 4.1 & 7.4 & 7.1 \\
\hline \multicolumn{5}{|l|}{ Education (\%) } \\
\hline Less than high school & 50.6 & 62.5 & 51.8 & 39.3 \\
\hline High school & 29.1 & 29.1 & 14.8 & 42.8 \\
\hline University & 20.2 & 8.3 & 33.3 & 17.8 \\
\hline Sedentary (min/day) & $507.2(84.9)$ & $548.8(80.2)$ & $507.6(79.1)$ & $471.3(80.1)$ \\
\hline LIPA (min/day) & $294.2(95)$ & $248.4(91.3)$ & $309.9(92.3)$ & $359.5(91.6)$ \\
\hline MVPA (min/day) & $22.9(20.3)$ & I I.3 (I I.8) & $32.8(23.1)$ & $23.2(18.3)$ \\
\hline Counts/day $(\times 1,000)$ & $237.4(124.5)$ & $160.8(86.5)$ & $283.7(131.6)$ & $259.0(118.1)$ \\
\hline Wearing time (h/day) & I3.7 (I.5) & $13.5(1.7)$ & I4.2 (I.2) & | $4.5(1.3)$ \\
\hline \multicolumn{5}{|l|}{ Valid days (\%) } \\
\hline 7 & 62 & 66.7 & 70.4 & 50.0 \\
\hline$\leq 3$ & 2.5 & 4.1 & 0 & 3.5 \\
\hline
\end{tabular}

Note: Data expressed as mean (SD) or percentages.

Abbreviations: CVD, cardiovascular disease; BMI, body mass index; LIPA, light-intensity physical activity; MVPA, moderate-vigorous physical activity.

time was replaced with an equal amount of light-intensity PA. For cancer mortality, the risk reduction of replacing 30 minutes of sedentary time with light-intensity PA was $14 \%$ $(P=0.059)$. Replacing sedentary time with MVPA resulted in survival advantages for CVD mortality. Risk reductions were $38 \%$ for replacing 10 minutes/day and $77 \%$ for replacing 30 minutes/day. No statistically significant reductions in all-cause or cancer mortality were found when sedentary time was replaced with MVPA.

\section{Discussion}

We used isotemporal substitution models to estimate mortality risk after 15 years in the ABC study sample, the first national population-based study collecting objectively assessed sedentary time and PA. Our findings suggest that if sedentary time is replaced with light-intensity PA, the risk of mortality from any cause, as well as from CVD, is reduced. Replacing sedentary time with MVPA decreased the risk of mortality from CVD. No survival benefits were found for mortality from cancer, although the results from replacing sedentary time with light-intensity PA were close to statistical significance.

The advantage of the isotemporal model is that it allows the comparison of substitution of a fixed time of one PA behavior for the same time engaged in another PA behavior.
This analysis method can help to answer the public health question of how to spend our discretionary time for health. To the best of our knowledge, to date only three previous studies ${ }^{15,16,23}$ have investigated mortality risks of objectively assessed sedentary time using isotemporal substitution. All of them analyzed data from the US National Health and Nutrition Examination Survey (NHANES) in 2003-2004 and 2005-2006, with a mean follow-up of 6.35-6.6 years. ${ }^{15,16,23}$ The results from these past studies showed $14 \%-20 \%$ risk reductions in all-cause mortality if 30 minutes/day of sedentary behavior was replaced with light-intensity $\mathrm{PA}^{15,23}$ or $18 \%$ lower risk if 60 minutes/day was replaced. ${ }^{16}$ The risk reduction in our sample was slightly lower, but overall these results provide consistent evidence that light-intensity PA may be more beneficial for health than previously known. Therefore, the effect of substituting sedentary time with light-intensity PA can be substantial and clinically relevant if the amount of time exchanged is sufficient.

In contrast to the results from the NHANES cohort ${ }^{15,16,23}$ and contrary to our expectations, we did not find statistically significant risk reductions for all-cause mortality from substituting sedentary time with MVPA, although for CVDmortality risk the survival benefits were large if sedentary time was replaced by MVPA. A daily increase of 10 minutes of MVPA, equivalent to approximately half the recommended 


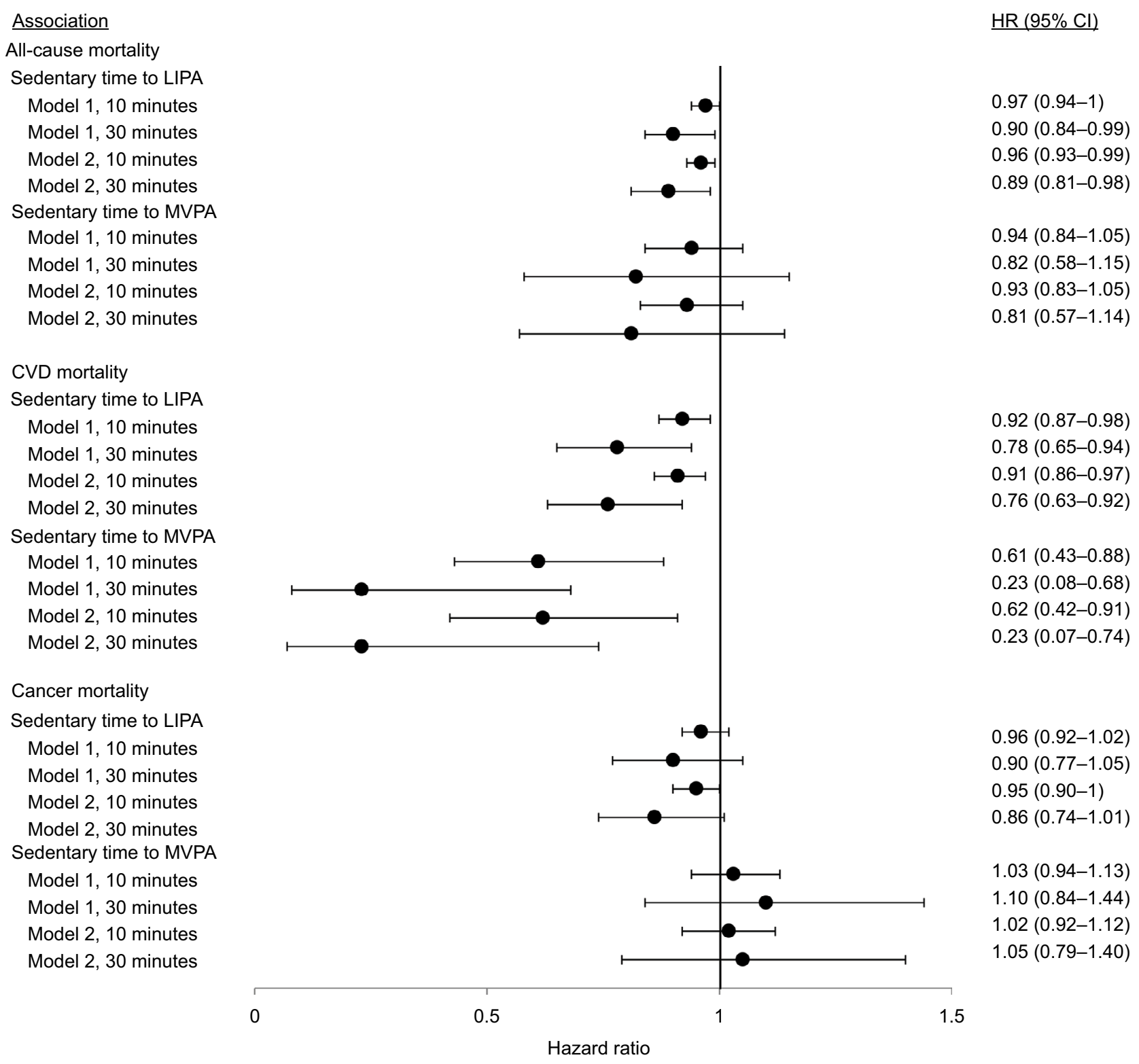

Figure I All-cause mortality, cardiovascular disease (CVD) mortality, and cancer mortality.

Notes: Associated with replacement of 10 and 30 minutes of sedentary time with an equal amount of time spent in light-intensity physical activity (LIPA) or moderatevigorous physical activity (MVPA). Model I comprises LIPA, MVPA, and total accelerometer-wearing time, stratified by sex and age tertiles. Model 2 is additionally adjusted for education, smoking, and presence of hypertension, heart disease, cancer, and diabetes at baseline. Sedentary time was defined as $<100$ counts/minute, LIPA as I00-2,0I 9 counts/minute, and MVPA as $\geq 2,020$ counts/minute.

Abbreviation: $\mathrm{HR}$, hazard ratio.

dose of PA for health, ${ }^{24,25}$ reduced the risk of CVD mortality by almost $40 \%$. A possible explanation for the inconsistency in risk reductions of all-cause mortality from light-intensity PA and MVPA might be that they depend on different physiological mechanisms. There is an ongoing debate on whether sedentary behavior is associated with increased mortality through distinct mechanisms biologically independent from the consequences of insufficient MVPA. ${ }^{10,11}$ It is also possible that a larger sample is needed to show significant results for replacement of sedentary behavior with MVPA, due to the skewed distribution of MVPA in our data set or possible interindividual responses to MVPA. In addition, the lack of association between cancer and MVPA influenced the outcome for all-cause mortality. Current evidence has found the associations between various sedentary behavior measures and all-cause or CVD mortality to be fairly consistent, whereas findings for cancer mortality are inconsistent. ${ }^{11,26}$ Although PA plays an important role at all points of interest for cancer survival, the mechanisms by which PA affects cancer mortality are complex and may differ by cancer site. ${ }^{26}$ Similarly to our results, Schmid et al ${ }^{15}$ found small or nonsignificant survival effects when sedentary behavior was 
replaced with PA. Larger studies may be needed, allowing analysis by both different PA intensities and different types of cancers, to investigate these associations further.

It is essential to acknowledge that isotemporal substitution models reflect only the theoretical effects of replacing one activity for another, rather than actual changes in behavior. Studies examining actual behavioral changes are needed to confirm our findings. Furthermore, the choice of the length of the replaced bout does not affect the statistical significance of the results, only the strength of the associations. We chose to analyze the replacement of both 10- and 30-minute bouts to be able to present results that would be useful from a behavioral counseling perspective. If sedentary time is replaced with light-intensity PA, a bout of at least 30 minutes may be both relevant and achievable, but if sedentary time is substituted with MVPA, a 10-minute replacement may be more realistic for many individuals. ${ }^{27}$

Our study has a number of strengths. We analyzed data from the first population-based cohort using accelerometry, enabling 15 years' follow-up. To the best of our knowledge, this is the longest follow-up of objectively measured sedentary behavior and PA in a nationally representative sample. In comparison with earlier published research, ${ }^{15,16}$ we had $>7$ years longer follow-up. The high accelerometer-wearing time compliance and the reliable register data on mortality and cause of death are also important strengths. Additionally, our choice to estimate the mortality benefits for both a 10-minute and a 30-minute reduction in sedentary time makes our findings easy to translate into clinical advice, as well as public health messages.

Our study also has some limitations. Compared to other population-based studies, our sample size was relatively small, and all background characteristics were self-reported. The activity data of our study were not assessed repeatedly during follow-up; therefore, we do not have information about changes in sedentary behavior or PA habits over time that may have influenced the observed associations. However, analyses of an ABC study subsample ${ }^{28}$ found that total PA remained unchanged from 2001 to 2008, suggesting that potential changes are small and will not influence the results on a group level.

Despite the advantages of objective assessment of PA using accelerometry, there are some limitations with this method. First, accelerometers do not capture all PA, such as upper body movements, biking, and swimming. Second, the ActiGraph records body movement and not postures. Consequently, periods of standing still may be misclassified as sedentary time. A third limitation is the challenges associated with translation of activity counts into categories of different PA intensity, ${ }^{29,30}$ and the estimates of time spent in different intensity categories are highly sensitive to selected cutoff points. The cutoff point we chose for sedentary time ( $<100$ counts/minute) is the most frequently used in studies on older adults ${ }^{31}$ and has also been used in similar studies. ${ }^{15,16,23}$ Several cutoff points have been proposed for MVPA, ${ }^{31,32}$ and there is lack of consensus on cutoff points appropriate for large epidemiological studies. The use of cutoff points to determine MVPA may be most problematic for older adults, and specific, lower cutoff points have been suggested. ${ }^{31}$ We chose the $\geq 2,020$ counts/minute cutoff point, since it is equivalent to walking at $4 \mathrm{~km} / \mathrm{h},{ }^{33}$ which is achievable moderate intensity for most people. ${ }^{34,35}$ It is also an advantage that this cutoff point has previously been used in the NHANES data set, ${ }^{15,16,23}$ which facilitates comparisons. Matthews et al ${ }^{16,30}$ suggested the $\geq 760$ counts/minute cutoff point to be the most appropriate for MVPA; however, we have previously shown that it is very likely to overestimate time in MVPA in our sample. ${ }^{8}$

Even though we adjusted for potentially important confounders, as any observational study, our results may be subject to unmeasured confounding. For example, we did not have information on alcohol use or mobility limitations. Other potential confounders are modifiable lifestyle factors like dietary patterns and stress. Finally, the accelerometers were worn only during waking hours, and we did not have any reliable information on sleeping time. Recent research ${ }^{36}$ has shown that isotemporal models with a 24-hour approach, including sleeping time, may add important information to the associations explored in this study.

\section{Conclusion}

This study strengthens recent findings that light-intensity PA might be even more beneficial for health than previously found. If 30 minutes of daily sedentary time was replaced with light-intensity PA, the risk of all-cause mortality and CVD mortality was reduced by $11 \%$ and $24 \%$, respectively. Replacement of sedentary time with MVPA reduced CVD mortality, but not all-cause or cancer mortality. Our results support the view that the most relevant public health message may be to promote the benefits of light-intensity activities and reduced sedentary time, without downplaying the health effects of MVPA.

\section{Acknowledgments}

The original ABC study was funded by Stockholm County Council, the Swedish National Centre for Research in Sports, and the project ALPHA, which received funding from the European Union in the framework of the Public Health Pro- 
gramme (agreement 2006120). This specific study has been funded by a research grant from Folksam Insurance, Sweden.

\section{Disclosure}

The authors report no conflicts of interest in this work.

\section{References}

1. Wilmot EG, Edwardson CL, Achana FA, et al. Sedentary time in adults and the association with diabetes, cardiovascular disease and death: systematic review and meta-analysis. Diabetologia. 2012;55(11):2895-2905.

2. Thorp AA, Owen N, Neuhaus M, Dunstan DW. Sedentary behaviors and subsequent health outcomes in adults a systematic review of longitudinal studies, 1996-2011. Am J Prev Med. 2011;41(2):207-215.

3. Chau JY, Grunseit A, Midthjell K, et al. Sedentary behaviour and risk of mortality from all-causes and cardiometabolic diseases in adults: evidence from the HUNT3 population cohort. Br J Sports Med. 2015;49(11):737-742.

4. Koster A, Caserotti P, Patel KV, et al. Association of sedentary time with mortality independent of moderate to vigorous physical activity. PLoS One. 2012;7(6):e37696.

5. Buman MP, Winkler EA, Kurka JM, et al. Reallocating time to sleep, sedentary behaviors, or active behaviors: associations with cardiovascular disease risk biomarkers, NHANES 2005-2006. Am J Epidemiol. 2014;179(3):323-334.

6. Sedentary Behaviour Research Network. Letter to the editor: standardized use of the terms "sedentary" and "sedentary behaviours". Appl Physiol Nutr Metab. 2012;37(3):540-542.

7. Matthews CE, George SM, Moore SC, et al. Amount of time spent in sedentary behaviors and cause-specific mortality in US adults. Am J Clin Nutr. 2012;95(2):437-445.

8. Dohrn IM, Sjöström M, Kwak L, Oja P, Hagströmer M. Accelerometermeasured sedentary time and physical activity: a 15 year follow-up of mortality in a Swedish population-based cohort. J Sci Med Sport. Epub 2017 Nov 7.

9. Biswas A, Oh PI, Faulkner GE, et al. Sedentary time and its association with risk for disease incidence, mortality, and hospitalization in adults: a systematic review and meta-analysis. Ann Intern Med. 2015;162(2):123-132.

10. Ekelund U, Steene-Johannessen J, Brown WJ, et al. Does physical activity attenuate, or even eliminate, the detrimental association of sitting time with mortality? A harmonised meta-analysis of data from more than 1 million men and women. Lancet. 2016;388(10051):1302-1310.

11. Young DR, Hivert MF, Alhassan S, et al. Sedentary behavior and cardiovascular morbidity and mortality: a science advisory from the American Heart Association. Circulation. 2016;134(13):e262-e279.

12. Matthews CE, Moore SC, Sampson J, et al. Mortality Benefits for replacing sitting time with different physical activities. Med Sci Sports Exerc. 2015;47(9):1833-1840.

13. Hagströmer M, Troiano RP, Sjöström M, Berrigan D. Levels and patterns of objectively assessed physical activity: a comparison between Sweden and the United States. Am J Epidemiol. 2010;171(10):1055-1064.

14. Sallis JF, Saelens BE. Assessment of physical activity by self-report: status, limitations, and future directions. Res Q Exerc Sport. 2000;71 (Suppl 2):S1-S14.

15. Schmid D, Ricci C, Baumeister SE, Leitzmann MF. Replacing sedentary time with physical activity in relation to mortality. Med Sci Sports Exerc. 2016;48(7):1312-1319.
16. Matthews CE, Keadle SK, Troiano RP, et al. Accelerometer-measured dose-response for physical activity, sedentary time, and mortality in US adults. Am J Clin Nutr. 2016;104(5):1424-1432.

17. Mekary RA, Willett WC, Hu FB, Ding EL. Isotemporal substitution paradigm for physical activity epidemiology and weight change. $\mathrm{Am} \mathrm{J}$ Epidemiol. 2009;170(4):519-527.

18. Hagströmer M, Oja P, Sjöström M. Physical activity and inactivity in an adult population assessed by accelerometry. Med Sci Sports Exerc. 2007;39(9):1502-1508.

19. Hagströmer M, Kwak L, Oja P, Sjöström M. A 6 year longitudinal study of accelerometer-measured physical activity and sedentary time in Swedish adults. J Sci Med Sport. 2014;18(5):553-557.

20. Troiano RP, Berrigan D, Dodd KW, Mâsse LC, Tilert T, McDowell M. Physical activity in the United States measured by accelerometer. Med Sci Sports Exerc. 2008;40(1):181-188.

21. Matthews CE, Chen KY, Freedson PS, et al. Amount of time spent in sedentary behaviors in the United States, 2003-2004. Am J Epidemiol. 2008;167(7):875-881.

22. Grambsch PM. Goodness-of-fit and diagnostics for proportional hazards regression models. Cancer Treat Res. 1995;75:95-112.

23. Fishman EI, Steeves JA, Zipunnikov V, et al. Association between objectively measured physical activity and mortality in NHANES. Med Sci Sports Exerc. 2016;48(7):1303-1311.

24. Haskell WL, Lee IM, Pate RR, et al. Physical activity and public health: updated recommendation for adults from the American College of Sports Medicine and the American Heart Association. Circulation. 2007;116(9):1081-1093.

25. World Health Organization. Global Recommendations on Physical Activity for Health: 65 Years and Above. Geneva: WHO; 2011.

26. Brown JC, Winters-Stone K, Lee A, Schmitz KH. Cancer, physical activity, and exercise. Compr Physiol. 2012;2(4):2775-2809.

27. Prince SA, Saunders TJ, Gresty K, Reid RD. A comparison of the effectiveness of physical activity and sedentary behaviour interventions in reducing sedentary time in adults: a systematic review and meta-analysis of controlled trials. Obes Rev. 2014;15(11):905-919.

28. Hagströmer M, Kwak L, Oja P, Sjöström M. A 6 year longitudinal study of accelerometer-measured physical activity and sedentary time in Swedish adults. J Sci Med Sport. 2015;18(5):553-557.

29. Bassett DR Jr, Rowlands A, Trost SG. Calibration and validation of wearable monitors. Med Sci Sports Exerc. 2012;44(1 Suppl 1):S32-S38.

30. Matthews CE. Calibration of accelerometer output for adults. Med Sci Sports Exerc. 2005;37(11 Suppl):S512-S522.

31. Gorman E, Hanson HM, Yang PH, Khan KM, Liu-Ambrose T, Ashe MC. Accelerometry analysis of physical activity and sedentary behavior in older adults: a systematic review and data analysis. Eur Rev Aging Phys Act. 2014;11:35-49.

32. Keadle SK, Shiroma EJ, Freedson PS, Lee IM. Impact of accelerometer data processing decisions on the sample size, wear time and physical activity level of a large cohort study. BMC Public Health. 2014;14:1210.

33. John D, Tyo B, Bassett DR. Comparison of four ActiGraph accelerometers during walking and running. Med Sci Sports Exerc. 2010;42(2):368-374.

34. Bohannon RW, Andrews AW. Normal walking speed: a descriptive meta-analysis. Physiotherapy. 2011;97(3):182-189.

35. Ainsworth BE, Haskell WL, Herrmann SD, et al. 2011 Compendium of physical activities: a second update of codes and MET values. Med Sci Sports Exerc. 2011;43(8):1575-1581.

36. Xiao Q, Keadle SK, Hollenbeck AR, Matthews CE. Sleep duration and total and cause-specific mortality in a large US cohort: interrelationships with physical activity, sedentary behavior, and body mass index. Am J Epidemiol. 2014;180(10):997-1006. 


\section{Publish your work in this journal}

Clinical Epidemiology is an international, peer-reviewed, open access, online journal focusing on disease and drug epidemiology, identification of risk factors and screening procedures to develop optimal preventative initiatives and programs. Specific topics include: diagnosis, prognosis, treatment, screening, prevention, risk factor modification,

Submit your manuscript here: https://www.dovepress.com/clinical-epidemiology-journa systematic reviews, risk and safety of medical interventions, epidemiology and biostatistical methods, and evaluation of guidelines, translational medicine, health policies and economic evaluations. The manuscript management system is completely online and includes a very quick and fair peer-review system, which is all easy to use. 